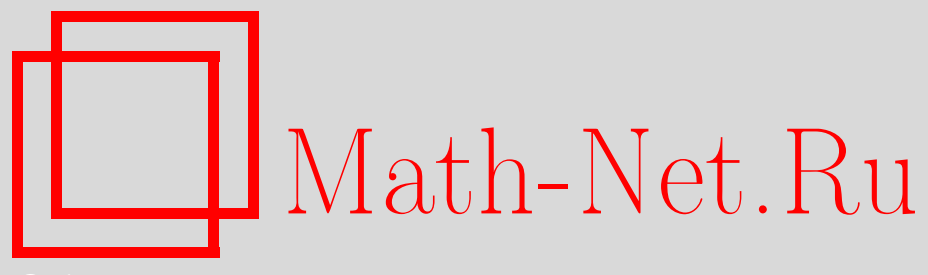

О. И. Мохов, Нелокальные гамильтоновы операторы гидродинамического типа с плоскими метриками, интегрируемые иерархии и уравнения ассоциативности, Функи. анализ и его прил., 2006, том 40, выпуск 1, 14-29

DOI: https://doi.org/10.4213/faa15

Использование Общероссийского математического портала Math-Net.Ru подразумевает, что вы прочитали и согласны с пользовательским соглашением

http://www. mathnet.ru/rus/agreement

Параметры загрузки:

IP: 3.89 .197 .203

26 апреля 2023 г., 15:45:48

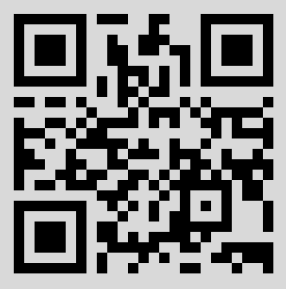




\title{
Нелокальные гамильтоновы операторы гидродинамического типа с плоскими метриками, интегрируемые иерархии и уравнения ассоциативности*
}

\author{
(C) 2006. О. И. Мохов
}

\section{§1. Введение}

В данной статье мы решаем задачу описания всех нелокальных гамильтоновых операторов гидродинамического типа с плоскими метриками и устанавливаем, что этот нетривиальный специальный класс гамильтоновых операторов тесно связан с уравнениями ассоциативности двумерных топологических квантовых теорий поля и теорией фробениусовых многообразий. В ней показано, что гамильтоновы операторы этого класса представляют особый интерес и по многим другим причинам. В частности, мы доказываем, что любой такой гамильтонов оператор всегда определяет интегрируемые структурные потоки (системы гидродинамического типа), всегда задает нетривиальный пучок согласованных гамильтоновых операторов и порождает интегрируемые иерархии гидродинамического типа. Аффиноры любого такого гамильтонова оператора порождают некоторые специальные интегралы в инволюции. Нелинейные системы, описывающие интегралы в инволюции, представляют большой самостоятельный интерес, и мы посвятим системам интегралов в инволюции в теории поля и их связям с уравнениями ассоциативности отдельную статью. Уравнения ассоциативности двумерных топологических квантовых теорий поля (уравнения Виттена-Дейкграфа-Верлинде-Верлинде и Дубровина) описывают важный специальный класс интегралов в инволюции и специальный класс нелокальных гамильтоновых операторов гидродинамического типа с плоскими метриками. Доказывается, что локально всякое $N$-мерное фробениусово многообразие представляется некоторым специальным плоским $N$-мерным подмногообразием с плоской нормальной связностью в $2 N$-мерном псевдоевклидовом пространстве, определяемым однозначно с точностью до движений. Свойствам этой конструкции и этого специального класса плоских подмногообразий с плоской нормальной связностью, отвечающего фробениусовым многообразиям, будет посвящена другая работа.

Напомним, что в [1] в связи с насущными потребностями гамильтоновой теории систем гидродинамического типа (см. также $[2,3])$ Ферапонтовым были изучены общие нелокальные гамильтоновы операторы гидродинамического типа,

* Работа выполнена при финансовой поддержке Фонда содействия отечественной науке, Российского фонда фундаментальных исследований (гранты № 03-01-00782 и № 05-01-00170) и Программы поддержки ведущих научных школ (грант №2185.2003.1). 
а именно гамильтоновы операторы вида

$$
\begin{aligned}
P^{i j}=g^{i j} & (u(x)) \frac{d}{d x}+b_{k}^{i j}(u(x)) u_{x}^{k} \\
& +\sum_{n=1}^{L} \varepsilon^{n}\left(w_{n}\right)_{k}^{i}(u(x)) u_{x}^{k}\left(\frac{d}{d x}\right)^{-1} \circ\left(w_{n}\right)_{s}^{j}(u(x)) u_{x}^{s},
\end{aligned}
$$

где $\operatorname{det}\left(g^{i j}(u)\right) \neq 0, \varepsilon^{n}= \pm 1,1 \leqslant n \leqslant L, u^{1}, \ldots, u^{N}$ - локальные координаты, $u=\left(u^{1}, \ldots, u^{N}\right), u^{i}(x), 1 \leqslant i \leqslant N,-$ функции (поля) одной независимой переменной $x$, а коэффициенты $g^{i j}(u), b_{k}^{i j}(u),\left(w_{n}\right)_{j}^{i}(u), 1 \leqslant i, j, k \leqslant N, 1 \leqslant n \leqslant L,-$ гладкие функции локальных координат.

Гамильтоновы операторы общего вида (1.1) (локальные и нелокальные) играют ключевую роль в гамильтоновой теории систем гидродинамического типа. Напомним, что оператор $P^{i j}$ называется гамилътоновым, если он определяет скобку Пуассона

$$
\{I, J\}=\int \frac{\delta I}{\delta u^{i}(x)} P^{i j} \frac{\delta J}{\delta u^{j}(x)} d x
$$

на произвольных функционалах $I$ и $J$ на пространстве полей $u^{i}(x)$, т. е. скобка (1.2) является кососимметричной и удовлетворяет тождеству Якоби. В [1] доказано, что оператор (1.1) является гамильтоновым тогда и только тогда, когда $g^{i j}(u)$ - симметричная (псевдориманова) контравариантная метрика и выполнены следующие соотношения:

1) $b_{k}^{i j}(u)=-g^{i s}(u) \Gamma_{s k}^{j}(u)$, где $\Gamma_{s k}^{j}(u)$ - риманова связность, порождаемая контравариантной метрикой $g^{i j}(u)$ (связность Леви-Чивиты);

2) $g^{i k}(u)\left(w_{n}\right)_{k}^{j}(u)=g^{j k}(u)\left(w_{n}\right)_{k}^{i}(u)$;

3) $\nabla_{k}\left(w_{n}\right)_{j}^{i}(u)=\nabla_{j}\left(w_{n}\right)_{k}^{i}(u)$, где $\nabla_{k}$ - ковариантная производная, порождаемая связностью Леви-Чивиты $\Gamma_{s k}^{j}(u)$ метрики $g^{i j}(u)$;

4) $R_{k l}^{i j}(u)=\sum_{n=1}^{L} \varepsilon^{n}\left(\left(w_{n}\right)_{l}^{i}(u)\left(w_{n}\right)_{k}^{j}(u)-\left(w_{n}\right)_{l}^{j}(u)\left(w_{n}\right)_{k}^{i}(u)\right)$, где $R_{k l}^{i j}(u)=$ $g^{i s}(u) R_{s k l}^{j}(u)$ - тензор римановой кривизны метрики $g^{i j}(u)$;

5) $\left[w_{n}(u), w_{m}(u)\right]=0$, т. е. семейство тензоров типа $(1,1)$ (аффбиноров) $\left(w_{n}\right)_{j}^{i}(u), 1 \leqslant n \leqslant L$, коммутативно.

Гамильтонов оператор вида (1.1) в точности соответствует $N$-мерной поверхности с плоской нормальной связностью, вложенной в псевдоевклидово пространство $E^{N+L}$. При этом ковариантная метрика $g_{i j}(u)$, для которой $g_{i s}(u) g^{s j}(u)=\delta_{i}^{j}$, является первой квадратичной формой, а аффиноры $w_{n}(u)$, $1 \leqslant n \leqslant L$, являются операторами Вайнгартена этой вложенной поверхности $\left(g_{i s}(u)\left(w_{n}\right)_{j}^{s}(u)\right.$ - соответствующие вторые квадратичные формы). При такой интерпретации соотношения 2)-4) являются уравнениями Гаусса-ПетерсонаКодацци для $N$-мерной поверхности с плоской нормальной связностью, вложенной в псевдоевклидово пространство $E^{N+L}$ [1]. Соотношения 5) эквивалентны уравнениям Риччи для этой вложенной поверхности.

Учитывая дальнейшие приложения к произвольным фробениусовым многообразиям, мы предпочитаем рассматривать общие нелокальные гамильтоновы 
операторы гидродинамического типа в виде

$$
\begin{aligned}
P^{i j}=g^{i j} & (u(x)) \frac{d}{d x}+b_{k}^{i j}(u(x)) u_{x}^{k} \\
& +\sum_{m=1}^{L} \sum_{n=1}^{L} \mu^{m n}\left(w_{m}\right)_{k}^{i}(u(x)) u_{x}^{k}\left(\frac{d}{d x}\right)^{-1} \circ\left(w_{n}\right)_{s}^{j}(u(x)) u_{x}^{s},
\end{aligned}
$$

где $\operatorname{det}\left(g^{i j}(u)\right) \neq 0$, а $\mu^{m n}$ - произвольная невырожденная симметричная постоянная матрица. Рассматривая линейные преобразования в векторном пространстве аффиноров $w_{n}(u), 1 \leqslant n \leqslant L$, т. е. заменяя в (1.3) все аффиноры $w_{n}(u)$ на $c_{n}^{l} \widetilde{w}_{l}(u)$, где $c_{n}^{l}$ - произвольная невырожденная постоянная матрица, любой оператор вида (1.3) можно привести к виду (1.1) и, обратно, любой оператор вида (1.3) может быть получен из некоторого оператора вида (1.1) линейной заменой аффиноров вида $w_{n}(u)=c_{n}^{l} \widetilde{w}_{l}(u)$. Эти преобразования из всех условий гамильтоновости 1)-5) оператора (1.1) изменяют только условие на тензор римановой кривизны метрики (условие 4)), которое для оператора (1.3) принимает вид

$$
R_{k l}^{i j}(u)=\sum_{m=1}^{L} \sum_{n=1}^{L} \mu^{m n}\left(\left(w_{m}\right)_{l}^{i}(u)\left(w_{n}\right)_{k}^{j}(u)-\left(w_{m}\right)_{l}^{j}(u)\left(w_{n}\right)_{k}^{i}(u)\right) ;
$$

все остальные условия гамильтоновости не изменяются.

Лемма 1.1. Оператор (1.3) является гамильтоновым тогда и только тогда, когда его коэффищиенты удовлетворяют соотношениям

$$
\begin{aligned}
& g^{i j}=g^{j i}, \\
& \frac{\partial g^{i j}}{\partial u^{k}}=b_{k}^{i j}+b_{k}^{j i}, \\
& g^{i s} b_{s}^{j k}=g^{j s} b_{s}^{i k}, \\
& g^{i s}\left(w_{n}\right)_{s}^{j}=g^{j s}\left(w_{n}\right)_{s}^{i}, \\
& \left(w_{n}\right)_{s}^{i}\left(w_{m}\right)_{j}^{s}=\left(w_{m}\right)_{s}^{i}\left(w_{n}\right)_{j}^{s} \text {, } \\
& g^{i s} g^{j r} \frac{\partial\left(w_{n}\right)_{r}^{k}}{\partial u^{s}}-g^{j r} b_{s}^{i k}\left(w_{n}\right)_{r}^{s}=g^{j s} g^{i r} \frac{\partial\left(w_{n}\right)_{r}^{k}}{\partial u^{s}}-g^{i r} b_{s}^{j k}\left(w_{n}\right)_{r}^{s}, \\
& g^{i s}\left(\frac{\partial b_{s}^{j k}}{\partial u^{r}}-\frac{\partial b_{r}^{j k}}{\partial u^{s}}\right)+b_{s}^{i j} b_{r}^{s k}-b_{s}^{i k} b_{r}^{s j}=\sum_{m=1}^{L} \sum_{n=1}^{L} \mu^{m n} g^{i s}\left(\left(w_{m}\right)_{r}^{j}\left(w_{n}\right)_{s}^{k}-\left(w_{m}\right)_{s}^{j}\left(w_{n}\right)_{r}^{k}\right) \text {. }
\end{aligned}
$$

\section{§2. Пучок гамильтоновых операторов}

Рассмотрим важный частный случай нелокальных гамильтоновых операторов вида (1.3), когда метрика $g^{i j}(u)$ плоская. Напомним, что любая плоская метрика однозначно определяет локальный гамильтонов оператор гидродинамического типа (т. е. гамильтонов оператор вида (1.3) с нулевыми аффинорами) 
- гамильтонов оператор Дубровина-Новикова [2]. В данной работе мы доказываем, что для любой плоской метрики имеется и замечательный класс нелокальных гамильтоновых операторов гидродинамического типа с этой плоской метрикой и нетривиальными аффинорами, причем эти операторы имеют важные приложения в теории фробениусовых многообразий и интегрируемых иерархий. Сразу отметим одно важное свойство нелокальных гамильтоновых операторов гидродинамического типа с плоскими метриками. Напомним, что два гамильтоновых оператора называются согласованными, если все линейные комбинации этих гамильтоновых операторов также являются гамильтоновыми операторами [4], т. е. они образуют пучок гамильтоновых операторов.

ЛЕмма 2.1. Метрика $g^{i j}(u)$ гамилътонова оператора вида (1.3) является плоской тогда и только тогда, когда этот оператор определяет пучок согласованных гамильтоновых операторов

$$
\begin{aligned}
P_{\lambda_{1}, \lambda_{2}}^{i j}=\lambda_{1} & \left(g^{i j}(u(x)) \frac{d}{d x}+b_{k}^{i j}(u(x)) u_{x}^{k}\right) \\
& +\lambda_{2} \sum_{m=1}^{L} \sum_{n=1}^{L} \mu^{m n}\left(w_{m}\right)_{k}^{i}(u(x)) u_{x}^{k}\left(\frac{d}{d x}\right)^{-1} \circ\left(w_{n}\right)_{s}^{j}(u(x)) u_{x}^{s},
\end{aligned}
$$

где $\lambda_{1}$ и $\lambda_{2}$ - произвольные константы.

Действительно, если оператор (1.3) является гамильтоновым, то для него выполнены соотношения (1.4)-(1.10). В этом случае для оператора (2.1) соотношения (1.4)-(1.9) всегда выполнены для любых констант $\lambda_{1}$ и $\lambda_{2}$, а соотношение (1.10) выполняется для любых констант $\lambda_{1}$ и $\lambda_{2}$ тогда и только тогда, когда тождественно равны нулю левая и правая части этого соотношения. Из соотношений (1.4)-(1.6) для гамильтонова оператора (1.3) следует, что тензор римановой кривизны метрики $g^{i j}(u)$ имеет вид

$$
R_{r}^{i j k}(u)=g^{i s}(u) R_{s r}^{j k}(u)=g^{i s}(u)\left(\frac{\partial b_{s}^{j k}}{\partial u^{r}}-\frac{\partial b_{r}^{j k}}{\partial u^{s}}\right)+b_{s}^{i j}(u) b_{r}^{s k}(u)-b_{s}^{i k}(u) b_{r}^{s j}(u) .
$$

Если метрика $g^{i j}(u)$ гамильтонова оператора вида (1.3) является плоской, т. е. $R_{r}^{i j k}(u)=0$, то соотношение (1.10) принимает вид

$$
\sum_{m=1}^{L} \sum_{n=1}^{L} \mu^{m n} g^{i s}\left(\left(w_{m}\right)_{r}^{j}(u)\left(w_{n}\right)_{s}^{k}(u)-\left(w_{m}\right)_{s}^{j}(u)\left(w_{n}\right)_{r}^{k}(u)\right)=0 .
$$

Таким образом, метрика $g^{i j}(u)$ гамильтонова оператора (1.3) является плоской тогда и только тогда, когда тождественно равны нулю левая и правая части соотношения (1.10) для гамильтонова оператора (1.3), а в этом случае тождественно равны нулю левая и правая части соотношения (1.10) и для оператора (2.1) для любых констант $\lambda_{1}$ и $\lambda_{2}$, т. е. мы получаем пучок согласованных гамильтоновых операторов (2.1). Отметим также, что для пучка гамильтоновых операторов $P_{\lambda_{1}, \lambda_{2}}^{i j}$, заданного формулой (2.1), из теоремы Дубровина-Новикова [2] для локального оператора $P_{1,0}^{i j}$ немедленно следует, что метрика $g^{i j}(u)$ является плоской. 
Таким образом, если метрика $g^{i j}(u)$ гамильтонова оператора вида (1.3) является плоской, то оператор

$$
P_{0,1}^{i j}=\sum_{m=1}^{L} \sum_{n=1}^{L} \mu^{m n}\left(w_{m}\right)_{k}^{i}(u(x)) u_{x}^{k}\left(\frac{d}{d x}\right)^{-1} \circ\left(w_{n}\right)_{s}^{j}(u(x)) u_{x}^{s}
$$

также является гамильтоновым оператором, получаемым вырождением $\lambda_{1} \rightarrow 0$, причем этот гамильтонов оператор в этом случае всегда согласован с локальным гамильтоновым оператором гидродинамического типа (оператором ДубровинаНовикова)

$$
P_{1,0}^{i j}=g^{i j}(u(x)) \frac{d}{d x}+b_{k}^{i j}(u(x)) u_{x}^{k} .
$$

Согласованные гамильтоновы операторы (2.3) и (2.4) порождают интегрируемые бигамильтоновы иерархии. Мы построим эти интегрируемые иерархии далее в данной статье.

\section{§3. Интегрируемость структурных потоков}

Напомним, что системы гидродинамического типа

$$
u_{t_{n}}^{i}=\left(w_{n}\right)_{j}^{i}(u) u_{x}^{j}, \quad 1 \leqslant n \leqslant L,
$$

называются структурными потоками нелокального гамильтонова оператора гидродинамического типа (1.3) (см. [1,5]).

ЛЕмма 3.1. Все структурные потоки (3.1) любого нелокального гамильтонова оператора гидродинамического типа с плоской метрикой являются коммутирующими интегрируемыми бигамилътоновыми системами гидродинамического типа.

Как доказано Мальцевым и Новиковым в [5] (см. также [1]), структурные потоки любого нелокального гамильтонова оператора гидродинамического типа (1.3) являются гамильтоновыми относительно этого гамильтонова оператора. Рассмотрим произвольный нелокальный гамильтонов оператор гидродинамического типа (1.3) с плоской метрикой $g^{i j}(u)$ и отвечающий этому гамильтоновому оператору пучок согласованных гамильтоновых операторов (2.1). Соответствующие структурные потоки обязаны быть гамильтоновыми относительно любого из операторов гамильтонова пучка (2.1) и, следовательно, являются интегрируемыми бигамильтоновыми системами.

\section{§4. Описание нелокальных гамильтоновых операторов гидродинамического типа с плоскими метриками}

Опишем все нелокальные гамильтоновы операторы гидродинамического типа с плоскими метриками. Вид гамильтонова оператора (1.3) инвариантен относительно локальных замен координат, при этом все коэффициенты оператора преобразуются как соответствующие дифференциально-геометрические объекты. Так как метрика плоская, то существуют локальные координаты, в которых метрика приводится к постоянному виду $\eta^{i j}, \eta^{i j}=\mathrm{const}, \operatorname{det} \eta^{i j} \neq 0, \eta^{i j}=\eta^{j i}$. 
В этих локальных координатах все коэффициенты связности Леви-Чивиты равны нулю и гамильтонов оператор имеет вид

$$
\widetilde{P}^{i j}=\eta^{i j} \frac{d}{d x}+\sum_{m=1}^{L} \sum_{n=1}^{L} \mu^{m n}\left(\widetilde{w}_{m}\right)_{k}^{i}(u(x)) u_{x}^{k}\left(\frac{d}{d x}\right)^{-1} \circ\left(\widetilde{w}_{n}\right)_{s}^{j}(u(x)) u_{x}^{s} .
$$

Теорема 4.1. Onератор (4.1), где $\eta^{i j}$ и $\mu^{m n}$ - произвольные невырожсденные симметричные постоянные матрицы, является гамильтоновым тогда $u$ только тогда, когда существуют функиии $\psi_{n}(u), 1 \leqslant n \leqslant L$, такие, что

$$
\left(\widetilde{w}_{n}\right)_{j}^{i}(u)=\eta^{i s} \frac{\partial^{2} \psi_{n}}{\partial u^{s} \partial u^{j}},
$$

причем выполняются следующие соотношения:

$$
\begin{gathered}
\sum_{m=1}^{N} \sum_{n=1}^{N} \eta^{m n} \frac{\partial^{2} \psi_{j}}{\partial u^{i} \partial u^{m}} \frac{\partial^{2} \psi_{k}}{\partial u^{n} \partial u^{l}}=\sum_{m=1}^{N} \sum_{n=1}^{N} \eta^{m n} \frac{\partial^{2} \psi_{k}}{\partial u^{i} \partial u^{m}} \frac{\partial^{2} \psi_{j}}{\partial u^{n} \partial u^{l}} \\
\sum_{m=1}^{L} \sum_{n=1}^{L} \mu^{m n} \frac{\partial^{2} \psi_{m}}{\partial u^{i} \partial u^{j}} \frac{\partial^{2} \psi_{n}}{\partial u^{k} \partial u^{l}}=\sum_{m=1}^{L} \sum_{n=1}^{L} \mu^{m n} \frac{\partial^{2} \psi_{m}}{\partial u^{i} \partial u^{k}} \frac{\partial^{2} \psi_{n}}{\partial u^{j} \partial u^{l}}
\end{gathered}
$$

Для оператора вида (4.1) соотношения (1.4)-(1.6) выполняются автоматически, а соотношение (1.9) имеет вид

$$
\frac{\partial\left(\widetilde{w}_{n}\right)_{r}^{k}}{\partial u^{s}}=\frac{\partial\left(\widetilde{w}_{n}\right)_{s}^{k}}{\partial u^{r}}
$$

и, следовательно, локально существуют функции $\varphi_{n}^{i}(u), 1 \leqslant i \leqslant N, 1 \leqslant n \leqslant L$, такие, что

$$
\left(\widetilde{w}_{n}\right)_{j}^{i}(u)=\frac{\partial \varphi_{n}^{i}}{\partial u^{j}} .
$$

Соотношение (1.7) принимает вид

$$
\eta^{i s} \frac{\partial \varphi_{n}^{j}}{\partial u^{s}}=\eta^{j s} \frac{\partial \varphi_{n}^{i}}{\partial u^{s}}
$$

или, эквивалентно,

$$
\frac{\partial\left(\eta_{i s} \varphi_{n}^{s}\right)}{\partial u^{j}}=\frac{\partial\left(\eta_{j s} \varphi_{n}^{s}\right)}{\partial u^{i}}
$$

где $\eta_{i j}$ - матрица, обратная матрице $\eta^{i j}, \eta_{i s} \eta^{s j}=\delta_{i}^{j}$. Из соотношения (4.8) следует, что локально существуют функции $\psi_{n}(u), 1 \leqslant n \leqslant L$, такие, что

$$
\eta_{i s} \varphi_{n}^{s}=\frac{\partial \psi_{n}}{\partial u^{i}}
$$

Таким образом,

$$
\varphi_{n}^{i}=\eta^{i s} \frac{\partial \psi_{n}}{\partial u^{s}}, \quad\left(\widetilde{w}_{n}\right)_{j}^{i}(u)=\eta^{i s} \frac{\partial^{2} \psi_{n}}{\partial u^{s} \partial u^{j}} .
$$

При этом соотношения (1.8) и (1.10) приобретают вид (4.3) и (4.4) соответственно. 
СлЕДСТВИЕ 4.1. Если бункиии $\psi_{n}(u), 1 \leqslant n \leqslant L$, являются решением системы уравнениц (4.3), (4.4), то системы гидродинамического типа

$$
u_{t_{n}}^{i}=\eta^{i s} \frac{\partial^{2} \psi_{n}}{\partial u^{s} \partial u^{j}} u_{x}^{j}, \quad 1 \leqslant n \leqslant L,
$$

являются коммутирующими интегрируемыми бигамильтоновыми системами гидродинамического типа. Кроме того, в этом случае оператор

$$
M_{1}^{i j}=\sum_{m=1}^{L} \sum_{n=1}^{L} \mu^{m n} \eta^{i p} \eta^{j r} \frac{\partial^{2} \psi_{m}}{\partial u^{p} \partial u^{k}} u_{x}^{k}\left(\frac{d}{d x}\right)^{-1} \circ \frac{\partial^{2} \psi_{n}}{\partial u^{r} \partial u^{s}} u_{x}^{s}
$$

такэе является гамильтоновым оператором, причем он согласован с постоянным гамильтоновым оператором

$$
M_{2}^{i j}=\eta^{i j} \frac{d}{d x}
$$

В произвольных локальных координатах получаем следующее описание всех нелокальных гамильтоновых операторов гидродинамического типа с плоскими метриками.

Tеорема 4.2. Oператор (1.3) с плоской метрикой $g^{i j}(u)$ является гамильтоновим тогда и толъко тогда, когда $b_{k}^{i j}(u)=-g^{i s}(u) \Gamma_{s k}^{j}(u)$, где $\Gamma_{s k}^{j}(u)-$ плоская связность, порожддаемая метрикой $g^{i j}(u)$, и локально существуют функции $\Phi_{n}(u), 1 \leqslant n \leqslant L$, такие, что

$$
\left(w_{n}\right)_{j}^{i}(u)=\nabla^{i} \nabla_{j} \Phi_{n}
$$

причем выполняются следующие соотношения:

$$
\begin{gathered}
\sum_{n=1}^{N} \nabla^{n} \nabla_{i} \Phi_{j} \nabla_{n} \nabla_{l} \Phi_{k}=\sum_{n=1}^{N} \nabla^{n} \nabla_{i} \Phi_{k} \nabla_{n} \nabla_{l} \Phi_{j}, \\
\sum_{m=1}^{L} \sum_{n=1}^{L} \mu^{m n} \nabla_{i} \nabla_{j} \Phi_{m} \nabla_{k} \nabla_{l} \Phi_{n}=\sum_{m=1}^{L} \sum_{n=1}^{L} \mu^{m n} \nabla_{i} \nabla_{k} \Phi_{m} \nabla_{j} \nabla_{l} \Phi_{n},
\end{gathered}
$$

где $\nabla_{i}$ - ковариантная производная, определяемая плоской связностъю $\Gamma_{j k}^{i}(u)$, порождаемой метрикой $g^{i j}(u), \nabla^{i}=g^{i s}(u) \nabla_{s}$. B частности, в этом случае onepamop

$$
\begin{aligned}
M_{\lambda_{1}, \lambda_{2}}^{i j}=\lambda_{1} & \left(g^{i j}(u(x)) \frac{d}{d x}-g^{i s}(u(x)) \Gamma_{s k}^{j}(u(x)) u_{x}^{k}\right) \\
& +\lambda_{2} \sum_{m=1}^{L} \sum_{n=1}^{L} \mu^{m n} \nabla^{i} \nabla_{k} \Phi_{m} u_{x}^{k}\left(\frac{d}{d x}\right)^{-1} \circ \nabla^{j} \nabla_{s} \Phi_{n} u_{x}^{s}
\end{aligned}
$$

является гамилътоновым оператором для любых констант $\lambda_{1}$ u $\lambda_{2}$, а системы гидродинамического типа

$$
u_{t_{n}}^{i}=\nabla^{i} \nabla_{j} \Phi_{n} u_{x}^{j}, \quad 1 \leqslant n \leqslant L,
$$

являются коммутирующими интегрируемыми бигамильтоновыми системами гидродинамического типа. 


\section{§5. Интегрируемые иерархии}

Рассмотрим оператор рекурсии

$$
R_{j}^{i}=\left(M_{1}\left(M_{2}\right)^{-1}\right)_{j}^{i}=\sum_{m=1}^{L} \sum_{n=1}^{L} \mu^{m n} \eta^{i p} \frac{\partial^{2} \psi_{m}}{\partial u^{p} \partial u^{k}} u_{x}^{k}\left(\frac{d}{d x}\right)^{-1} \circ \frac{\partial^{2} \psi_{n}}{\partial u^{j} \partial u^{s}} u_{x}^{s}\left(\frac{d}{d x}\right)^{-1},
$$

отвечающий согласованным гамильтоновым операторам (4.12) и (4.13). Применим построенный нами оператор рекурсии (5.1) к системе трансляций по $x$

$$
u_{t}^{i}=u_{x}^{i} .
$$

Любая система из иерархии

$$
u_{t_{s}}^{i}=\left(R^{s}\right)_{j}^{i} u_{x}^{j}, \quad s \in \mathbb{Z},
$$

является мультигамильтоновой интегрируемой системой гидродинамического типа. В частности, интегрируемой является любая система вида

$$
u_{t_{1}}^{i}=R_{j}^{i} u_{x}^{j},
$$

т. е. система

$$
u_{t_{1}}^{i}=\sum_{m=1}^{L} \sum_{n=1}^{L} \mu^{m n} \eta^{i p} \frac{\partial^{2} \psi_{m}}{\partial u^{p} \partial u^{k}} u_{x}^{k}\left(\frac{d}{d x}\right)^{-1} \circ \frac{\partial^{2} \psi_{n}}{\partial u^{j} \partial u^{s}} u^{j} u_{x}^{s} .
$$

Система гидродинамического типа (5.5) имеет следующий локальный вид:

$$
u_{t_{1}}^{i}=\sum_{m=1}^{L} \sum_{n=1}^{L} \mu^{m n} \eta^{i p} F_{n}(u) \frac{\partial^{2} \psi_{m}}{\partial u^{p} \partial u^{k}} u_{x}^{k}, \quad F_{n}=\frac{\partial \psi_{n}}{\partial u^{j}} u^{j}-\psi_{n}, \quad \frac{\partial^{2} \psi_{n}}{\partial u^{j} \partial u^{s}} u^{j}=\frac{\partial F_{n}}{\partial u^{s}} .
$$

Эта система гидродинамического типа является бигамильтоновой по отношению к согласованным гамильтоновым операторам (4.12) и (4.13):

$$
\begin{gathered}
u_{t_{1}}^{i}=\sum_{m=1}^{L} \sum_{n=1}^{L} \mu^{m n} \eta^{i p} \eta^{j r} \frac{\partial^{2} \psi_{m}}{\partial u^{p} \partial u^{k}} u_{x}^{k}\left(\frac{d}{d x}\right)^{-1}\left(\frac{\partial^{2} \psi_{n}}{\partial u^{r} \partial u^{s}} u_{x}^{s} \frac{\delta H_{1}}{\delta u^{j}(x)}\right), \\
H_{1}=\int h_{1}(u(x)) d x, \quad h_{1}(u(x))=\frac{1}{2} \eta_{i j} u^{i}(x) u^{j}(x), \\
u_{t_{1}}^{i}=\eta^{i j} \frac{d}{d x} \frac{\delta H_{2}}{\delta u^{j}(x)}, \quad H_{2}=\int h_{2}(u(x)) d x,
\end{gathered}
$$

так как в нашем случае локально всегда существует функция $h_{2}(u)$, такая, что

$$
\sum_{m=1}^{L} \sum_{n=1}^{L} \mu^{m n} \frac{\partial^{2} \psi_{m}}{\partial u^{j} \partial u^{k}} F_{n}(u)=\frac{\partial^{2} h_{2}}{\partial u^{j} \partial u^{k}} .
$$


Действительно, мы имеем

$$
\begin{aligned}
& \frac{\partial}{\partial u^{i}}\left(\sum_{m=1}^{L} \sum_{n=1}^{L} \mu^{m n} \frac{\partial^{2} \psi_{m}}{\partial u^{j} \partial u^{k}} F_{n}(u)\right) \\
& =\sum_{m=1}^{L} \sum_{n=1}^{L} \mu^{m n} \frac{\partial^{3} \psi_{m}}{\partial u^{i} \partial u^{j} \partial u^{k}} F_{n}(u)+\sum_{m=1}^{L} \sum_{n=1}^{L} \mu^{m n} \frac{\partial^{2} \psi_{m}}{\partial u^{j} \partial u^{k}} \frac{\partial F_{n}}{\partial u^{i}} \\
& =\sum_{m=1}^{L} \sum_{n=1}^{L} \mu^{m n} \frac{\partial^{3} \psi_{m}}{\partial u^{i} \partial u^{j} \partial u^{k}} F_{n}(u)+\sum_{m=1}^{L} \sum_{n=1}^{L} \mu^{m n} \frac{\partial^{2} \psi_{m}}{\partial u^{j} \partial u^{k}} \frac{\partial^{2} \psi_{n}}{\partial u^{i} \partial u^{s}} u^{s} \\
& =\sum_{m=1}^{L} \sum_{n=1}^{L} \mu^{m n} \frac{\partial^{3} \psi_{m}}{\partial u^{i} \partial u^{j} \partial u^{k}} F_{n}(u)+\sum_{m=1}^{L} \sum_{n=1}^{L} \mu^{m n} \frac{\partial^{2} \psi_{m}}{\partial u^{j} \partial u^{i}} \frac{\partial^{2} \psi_{n}}{\partial u^{k} \partial u^{s}} u^{s},
\end{aligned}
$$

где использовано соотношение (4.4). В силу симметрии по индексам $i$ и $j$ мы получаем

$$
\frac{\partial}{\partial u^{i}}\left(\sum_{m=1}^{L} \sum_{n=1}^{L} \mu^{m n} \frac{\partial^{2} \psi_{m}}{\partial u^{j} \partial u^{k}} F_{n}(u)\right)=\frac{\partial}{\partial u^{j}}\left(\sum_{m=1}^{L} \sum_{n=1}^{L} \mu^{m n} \frac{\partial^{2} \psi_{m}}{\partial u^{i} \partial u^{k}} F_{n}(u)\right),
$$

т. е. локально существуют функции $a_{k}(u), 1 \leqslant k \leqslant N$, такие, что

$$
\sum_{m=1}^{L} \sum_{n=1}^{L} \mu^{m n} \frac{\partial^{2} \psi_{m}}{\partial u^{j} \partial u^{k}} F_{n}(u)=\frac{\partial a_{k}}{\partial u^{j}} .
$$

В силу симметрии по индексам $j$ и $k$ получаем

$$
\frac{\partial a_{k}}{\partial u^{j}}=\frac{\partial a_{j}}{\partial u^{k}}
$$

т. е. локально существует функция $h_{2}(u)$, такая, что

$$
a_{k}(u)=\frac{\partial h_{2}}{\partial u^{k}} \text {. }
$$

Таким образом,

$$
\sum_{m=1}^{L} \sum_{n=1}^{L} \mu^{m n} \frac{\partial^{2} \psi_{m}}{\partial u^{j} \partial u^{k}} F_{n}(u)=\frac{\partial a_{k}}{\partial u^{j}}=\frac{\partial^{2} h_{2}}{\partial u^{j} \partial u^{k}} .
$$

Рассмотрим следующее уравнение интегрируемой иерархии (5.3):

$$
\begin{aligned}
u_{t_{2}}^{i} & =\left(R^{2}\right)_{j}^{i} u_{x}^{j} \\
& =\sum_{m=1}^{L} \sum_{n=1}^{L} \mu^{m n} \eta^{i p} \frac{\partial^{2} \psi_{m}}{\partial u^{p} \partial u^{k}} u_{x}^{k}\left(\frac{d}{d x}\right)^{-1} \circ \frac{\partial^{2} \psi_{n}}{\partial u^{j} \partial u^{s}} u_{x}^{s}\left(\frac{d}{d x}\right)^{-1} \circ \eta^{j r} \frac{d}{d x} \frac{\delta H_{2}}{\delta u^{r}(x)} \\
& =\sum_{m=1}^{L} \sum_{n=1}^{L} \mu^{m n} \eta^{i p} \frac{\partial^{2} \psi_{m}}{\partial u^{p} \partial u^{k}} u_{x}^{k}\left(\frac{d}{d x}\right)^{-1} \circ \frac{\partial^{2} \psi_{n}}{\partial u^{j} \partial u^{s}} u_{x}^{s} \eta^{j r} \frac{\partial h_{2}}{\partial u^{r}}
\end{aligned}
$$


Докажем, что в нашем случае всегда локально существуют функции $G_{n}(u)$, $1 \leqslant n \leqslant L$, такие, что

$$
\frac{\partial^{2} \psi_{n}}{\partial u^{j} \partial u^{s}} \eta^{j r} \frac{\partial h_{2}}{\partial u^{r}}=\frac{\partial G_{n}}{\partial u^{s}} .
$$

Действительно, мы имеем

$$
\begin{gathered}
\frac{\partial}{\partial u^{p}}\left(\frac{\partial^{2} \psi_{n}}{\partial u^{j} \partial u^{s}} \eta^{j r} \frac{\partial h_{2}}{\partial u^{r}}\right)=\frac{\partial^{3} \psi_{n}}{\partial u^{j} \partial u^{s} \partial u^{p}} \eta^{j r} \frac{\partial h_{2}}{\partial u^{r}}+\frac{\partial^{2} \psi_{n}}{\partial u^{j} \partial u^{s}} \eta^{j r} \frac{\partial^{2} h_{2}}{\partial u^{r} \partial u^{p}} \\
\quad=\frac{\partial^{3} \psi_{n}}{\partial u^{j} \partial u^{s} \partial u^{p}} \eta^{j r} \frac{\partial h_{2}}{\partial u^{r}}+\frac{\partial^{2} \psi_{n}}{\partial u^{j} \partial u^{s}} \eta^{j r}\left(\sum_{k=1}^{L} \sum_{l=1}^{L} \mu^{k l} \frac{\partial^{2} \psi_{k}}{\partial u^{r} \partial u^{p}} F_{l}(u)\right) \\
=\frac{\partial^{3} \psi_{n}}{\partial u^{j} \partial u^{s} \partial u^{p}} \eta^{j r} \frac{\partial h_{2}}{\partial u^{r}}+\sum_{k=1}^{L} \sum_{l=1}^{L} \mu^{k l} \eta^{j r} \frac{\partial^{2} \psi_{n}}{\partial u^{s} \partial u^{j}} \frac{\partial^{2} \psi_{k}}{\partial u^{r} \partial u^{p}} F_{l}(u) \\
=\frac{\partial^{3} \psi_{n}}{\partial u^{j} \partial u^{s} \partial u^{p}} \eta^{j r} \frac{\partial h_{2}}{\partial u^{r}}+\sum_{k=1}^{L} \sum_{l=1}^{L} \mu^{k l} \eta^{j r} \frac{\partial^{2} \psi_{k}}{\partial u^{s} \partial u^{j}} \frac{\partial^{2} \psi_{n}}{\partial u^{r} \partial u^{p}} F_{l}(u) \\
=\frac{\partial^{3} \psi_{n}}{\partial u^{j} \partial u^{s} \partial u^{p}} \eta^{j r} \frac{\partial h_{2}}{\partial u^{r}}+\sum_{k=1}^{L} \sum_{l=1}^{L} \mu^{k l} \eta^{j r} \frac{\partial^{2} \psi_{k}}{\partial u^{s} \partial u^{r}} \frac{\partial^{2} \psi_{n}}{\partial u^{j} \partial u^{p}} F_{l}(u),
\end{gathered}
$$

где использованы соотношение (4.3) и симметричность матрицы $\eta^{j r}$. Таким образом, доказано, что рассматриваемое выражение симметрично по индексам $p$ и $s$, т. е.

$$
\frac{\partial}{\partial u^{p}}\left(\frac{\partial^{2} \psi_{n}}{\partial u^{j} \partial u^{s}} \eta^{j r} \frac{\partial h_{2}}{\partial u^{r}}\right)=\frac{\partial}{\partial u^{s}}\left(\frac{\partial^{2} \psi_{n}}{\partial u^{j} \partial u^{p}} \eta^{j r} \frac{\partial h_{2}}{\partial u^{r}}\right) .
$$

Следовательно, локально существуют функции $G_{n}(u), 1 \leqslant n \leqslant L$, такие, что выполняется соотношение (5.18), и, таким образом, доказано, что второй поток интегрируемой иерархии (5.3) имеет вид локальной системы гидродинамического типа

$$
u_{t_{2}}^{i}=\sum_{m=1}^{L} \sum_{n=1}^{L} \mu^{m n} \eta^{i p} G_{n}(u) \frac{\partial^{2} \psi_{m}}{\partial u^{p} \partial u^{k}} u_{x}^{k} .
$$

Повторяя в точности приведенные выше рассуждения, доказываем по индукции, что если функции $\psi_{n}(u), 1 \leqslant n \leqslant L$, являются решением системы уравнений $(4.3),(4.4)$, то для любого $s \geqslant 1$ и соответствующей функции $h_{s}(u(x))$ (начиная с функции $h_{1}(u(x))=\frac{1}{2} \eta_{i j} u^{i}(x) u^{j}(x)$ ) всегда локально существуют функции $F_{n}^{(s)}(u), 1 \leqslant n \leqslant L$, такие, что

$$
\frac{\partial^{2} \psi_{n}}{\partial u^{j} \partial u^{p}} \eta^{j r} \frac{\partial h_{s}}{\partial u^{r}}=\frac{\partial F_{n}^{(s)}}{\partial u^{p}}
$$

и всегда локально существует функция $h_{s+1}(u(x))$, такая, что

$$
\sum_{m=1}^{L} \sum_{n=1}^{L} \mu^{m n} \frac{\partial^{2} \psi_{m}}{\partial u^{j} \partial u^{k}} F_{n}^{(s)}(u)=\frac{\partial^{2} h_{s+1}}{\partial u^{j} \partial u^{k}} .
$$


Выше мы доказали, что это утверждение верно при $s=1$ (при этом, в частности, $\left.F_{n}^{(1)}=F_{n}, F_{n}^{(2)}=G_{n}\right)$. Точно так же доказывается, что если это утверждение верно для $s=K \geqslant 1$, то оно верно и для $s=K+1$ (см. (5.11)-(5.16) и $(5.19),(5.20))$. Таким образом, доказано, что для любого $s \geqslant 1$ соответствующий поток интегрируемой иерархии (5.3) имеет вид локальной системы гидродинамического типа

$$
u_{t_{s}}^{i}=\sum_{m=1}^{L} \sum_{n=1}^{L} \mu^{m n} \eta^{i p} F_{n}^{(s)}(u) \frac{\partial^{2} \psi_{m}}{\partial u^{p} \partial u^{k}} u_{x}^{k} .
$$

Все потоки иерархии (5.3) являются коммутирующими интегрируемыми бигамильтоновыми системами гидродинамического типа с бесконечным семейством локальных интегралов в инволюции относительно обеих скобок Пуассона:

$$
\begin{gathered}
u_{t_{s}}^{i}=M_{1}^{i j} \frac{\delta H_{s}}{\delta u^{j}(x)}=\left\{u^{i}, H_{s}\right\}_{1}, \quad H_{s}=\int h_{s}(u(x)) d x, \\
u_{t_{s}}^{i}=M_{2}^{i j} \frac{\delta H_{s+1}}{\delta u^{j}(x)}=\left\{u^{i}, H_{s+1}\right\}_{2}, \quad\left\{H_{p}, H_{r}\right\}_{1}=0, \quad\left\{H_{p}, H_{r}\right\}_{2}=0,
\end{gathered}
$$

причем плотности гамильтонианов $h_{s}(u(x))$ связаны рекуррентными соотношениями (5.22), (5.23), которые в нашем случае всегда разрешимы.

Пусть функции $\psi_{n}(u), 1 \leqslant n \leqslant L$, являются решением системы уравнений (4.3), (4.4), т. е., в частности, нелокальный оператор $M_{1}^{i j}$ вида (4.12) является гамильтоновым. Рассмотрим гамильтонову систему

$$
u_{t}^{i}=M_{1}^{i j} \frac{\delta H}{\delta u^{j}(x)}, \quad H=\int h(u(x)) d x,
$$

с произвольным гамильтонианом гидродинамического типа $H$. Как доказано Ферапонтовым в [1], гамильтонова система с нелокальным гамильтоновым оператором гидродинамического типа (1.1) и гамильтонианом гидродинамического типа является локальной тогда и только тогда, когда гамильтониан является интегралом структурных потоков гамильтонова оператора. Это утверждение верно и для гамильтоновых операторов вида (2.3), а также вообще для любых слабо нелокальных гамильтоновых операторов (см. [5]). Для построенных в данной работе нелокальных гамильтоновых операторов $M_{1}^{i j}$, задаваемых формулой (4.12), этого условия на гамильтонианы достаточно для интегрируемости, т. е. все локальные гамильтоновы системы (5.27) являются интегрируемыми бигамильтоновыми системами.

ЛЕмма 5.1. Система (5.27) является локальной тогда и только тогда, когда плотность гамильтониана $h(u(x))$ удовлетворяет уравнениям

$$
\frac{\partial^{2} \psi_{n}}{\partial u^{j} \partial u^{s}} \eta^{j r} \frac{\partial^{2} h}{\partial u^{r} \partial u^{p}}=\frac{\partial^{2} \psi_{n}}{\partial u^{j} \partial u^{p}} \eta^{j r} \frac{\partial^{2} h}{\partial u^{r} \partial u^{s}} .
$$

ТЕОрема 5.1. Если гамильтонова система (5.27) локальна, то она интегрируема.

Нелинейные уравнения вида (4.3) и (5.28) представляют самостоятельный интерес, они имеют важное значение и очень естественную интерпретацию. 
ЛЕмма 5.2. Нелинейные уравнения (4.3) эквивалентны условию, что интегралы

$$
\Psi_{n}=\int \psi_{n}(u(x)) d x, \quad 1 \leqslant n \leqslant L,
$$

находятся в инволюиии относительно скобки Пуассона, задаваемой постоянным гамильтоновым оператором $M_{2}^{i j}$ вида (4.13), m.е.

$$
\left\{\Psi_{n}, \Psi_{m}\right\}=0
$$

Аналогично, уравнения (5.28) эквивалентны условию

$$
\left\{\Psi_{n}, H\right\}=0, \quad H=\int h(u(x)) d x .
$$

Отметим, что уравнения (4.15) эквивалентны условию инволютивности $L$ интегралов относительно произвольной скобки Дубровина-Новикова (невырожденной локальной скобки Пуассона гидродинамического типа).

СлЕДСТВИЕ 5.1. Гамильтонова система (5.27) является локальной тогда и только тогда, когда она порождается семейством $L+1$ интегралов в инволюици относительно скобки Пуассона, задаваемой постоянным гамилътоновым оператором $M_{2}^{i j}$ вида (4.13), а именно

$$
\begin{gathered}
\Psi_{n}=\int \psi_{n}(u(x)) d x, \quad 1 \leqslant n \leqslant L, \\
H=\int h(u(x)) d x, \quad\left\{\Psi_{n}, \Psi_{m}\right\}=0, \quad\left\{\Psi_{n}, H\right\}=0 .
\end{gathered}
$$

При этом система (5.27) является интегрируемой бигамильтоновой системой.

Важный специальный класс интегралов в инволюции порождается уравнениями ассоциативности двумерной топологической квантовой теории поля.

ЛЕмма 5.3. Функиия $\Phi\left(u^{1}, \ldots, u^{N}\right)$ порождает семейство

$$
I_{n}=\int \frac{\partial \Phi}{\partial u^{n}}(u(x)) d x, \quad\left\{I_{n}, I_{m}\right\}=0, \quad 1 \leqslant n, m \leqslant N,
$$

$N$ интегралов в инволюции относительно скобки Пуассона, задаваемой постоянным гамильтоновым оператором $M_{2}^{i j}$ вида (4.13), тогда и только тогда, когда функиия $\Phi(u)$ является решением уравнений ассоциативности двумерной топологчческой квантовой теории поля

$$
\sum_{m=1}^{N} \sum_{n=1}^{N} \eta^{m n} \frac{\partial^{3} \Phi}{\partial u^{i} \partial u^{j} \partial u^{m}} \frac{\partial^{3} \Phi}{\partial u^{n} \partial u^{k} \partial u^{l}}=\sum_{m=1}^{N} \sum_{n=1}^{N} \eta^{m n} \frac{\partial^{3} \Phi}{\partial u^{i} \partial u^{k} \partial u^{m}} \frac{\partial^{3} \Phi}{\partial u^{n} \partial u^{j} \partial u^{l}} .
$$

\section{§6. Плоские подмногообразия с плоской нормальной связностью, гессианы и нелокальные скобки Пуассона}

Нелинейные уравнения (4.3) и (4.4), описывающие все нелокальные гамильтоновы операторы гидродинамического типа с плоскими метриками, в точности эквивалентны условиям, что в $(N+L)$-мерное псевдоевклидово пространство вложено плоское $N$-мерное подмногообразие с плоской нормальной связностью, 
с первой квадратичной формой $\eta_{i j} d u^{i} d u^{j}$ и вторыми квадратичными формами $\omega_{n}(u)$, задаваемыми гессианами $L$ функций $\psi_{n}(u), 1 \leqslant n \leqslant L$,

$$
\omega_{n}(u)=\frac{\partial^{2} \psi_{n}}{\partial u^{i} \partial u^{j}} d u^{i} d u^{j} .
$$

Уравнения (4.4) являются уравнениями Гаусса для этого случая, а уравнения (4.3) - уравнениями Риччи (уравнения Петерсона-Кодацци-Майнарди выполняются в этом случае автоматически). По теореме Петерсона-Бонне любое решение $\psi_{n}(u), 1 \leqslant n \leqslant L$, системы уравнений (4.3) и (4.4) определяет в $(N+L)$-мерном псевдоевклидовом пространстве единственное с точностью до движений $N$-мерное подмногообразие с плоской нормальной связностью, с первой квадратичной формой $\eta_{i j} d u^{i} d u^{j}$ и вторыми квадратичными формами $\omega_{n}(u)$, задаваемыми гессианами функций $\psi_{n}(u)$.

Рассмотрим произвольное $N$-мерное плоское подмногообразие с плоской нормальной связностью в $(N+L)$-мерном псевдоевклидовом пространстве. Пусть $g_{i j}(u) d u^{i} d u^{j}$ - первая квадратичная форма этого плоского подмногообразия, где $g_{i j}(u)$ - плоская метрика. Тогда справедливо такое следствие теории подмногообразий и теоремы Бонне:

Лемма 6.1. Локально существуют функции $\Phi_{n}(u), 1 \leqslant n \leqslant L$, такие, что вторые квадратичные формы рассматриваемого подмногообразия имеют вид

$$
\Omega_{n}(u)=\nabla_{i} \nabla_{j} \Phi_{n} d u^{i} d u^{j}
$$

где $\nabla_{i}$ - ковариантная производная, определяемая связностью Леви- Чивитъ метрики $g_{i j}(u)$. При этом функиии $\Phi_{n}(u), 1 \leqslant n \leqslant L$, удовлетворяют уравнениям Риччи (4.15) и уравнениям Гаусса (4.16) для подмногообразий (уравнения Петерсона-Кодации-Майнарди выполняются в этом случае автоматически). Любое решение системы уравнений (4.15) и (4.16) определяет в $(N+L)$-мерном псевдоевклидовом пространстве единственное с точностъю до двиюений $N$-мерное подмногообразие с плоской нормальной связностью, с первой квадратичной бормой $g_{i j}(u) d u^{i} d u^{j}$ и вторьми квадратичнымми бормами $\Omega_{n}(u)$ ви$\partial a(6.1)$.

\section{§7. Уравнения ассоциативности и нелокальные скобки Пуассона}

Покажем, что класс нелокальных гамильтоновых операторов гидродинамического типа с плоскими метриками нетривиален, и опишем богатое и важное семейство операторов этого класса, порождаемое уравнениями ассоциативности двумерной топологической квантовой теории поля.

Соотношения (4.3) и (4.4), хотя и существенно различаются, но очень похожи, и особенно интересен случай естественной редукции, при которой соотношения (4.3) и (4.4) просто совпадают. Такая редукция немедленно приводит к уравнениям ассоциативности двумерной топологической теории поля.

Действительно, соотношения (4.3) и (4.4) совпадают, если $L=N, \mu^{m n}=$ $\eta^{m n}$ (или, например, $\mu^{m n}=c \eta^{m n}$, где $c$ - произвольная ненулевая константа) и существует функция $\Phi(u)$, такая, что $\psi_{n}=\partial \Phi / \partial u^{n}$ для всех $n$; при этом оба соотношения (4.3) и (4.4) совпадают с уравнениями ассоциативности (5.34) двумерной топологической теории поля для потенциала $\Phi(u)$. 
Таким образом, любое решение $\Phi(u)$ уравнений ассоциативности (5.34), которые, как известно, совместны, интегрируемы методом обратной задачи рассеяния и обладают богатым множеством нетривиальных решений (см. [6]), определяет нелокальный гамильтонов оператор гидродинамического типа

$$
L^{i j}=\eta^{i j} \frac{d}{d x}+\sum_{m=1}^{N} \sum_{n=1}^{N} \eta^{m n} \eta^{i p} \eta^{j r} \frac{\partial^{3} \Phi}{\partial u^{p} \partial u^{m} \partial u^{k}} u_{x}^{k}\left(\frac{d}{d x}\right)^{-1} \circ \frac{\partial^{3} \Phi}{\partial u^{r} \partial u^{n} \partial u^{s}} u_{x}^{s}
$$

с плоской метрикой и, более того, пучок согласованных гамильтоновых операторов

$L_{\lambda_{1}, \lambda_{2}}^{i j}=\lambda_{1} \eta^{i j} \frac{d}{d x}+\lambda_{2} \sum_{m=1}^{N} \sum_{n=1}^{N} \eta^{m n} \eta^{i p} \eta^{j r} \frac{\partial^{3} \Phi}{\partial u^{p} \partial u^{m} \partial u^{k}} u_{x}^{k}\left(\frac{d}{d x}\right)^{-1} \circ \frac{\partial^{3} \Phi}{\partial u^{r} \partial u^{n} \partial u^{s}} u_{x}^{s}$,

где $\lambda_{1}$ и $\lambda_{2}$ - произвольные константы. В частности, если $\Phi(u)-$ произвольное решение уравнений ассоциативности (5.34), то оператор

$$
L_{0,1}^{i j}=\sum_{m=1}^{N} \sum_{n=1}^{N} \eta^{m n} \eta^{i p} \eta^{j r} \frac{\partial^{3} \Phi}{\partial u^{p} \partial u^{m} \partial u^{k}} u_{x}^{k}\left(\frac{d}{d x}\right)^{-1} \circ \frac{\partial^{3} \Phi}{\partial u^{r} \partial u^{n} \partial u^{s}} u_{x}^{s}
$$

является гамильтоновым оператором, согласованным с постоянным гамильтоновым оператором

$$
L_{1,0}^{i j}=\eta^{i j} \frac{d}{d x} .
$$

Таким образом, для любого решения уравнений ассоциативности (5.34) мы получаем соответствующие интегрируемые иерархии (см. §5).

Метрика $\eta^{i j}$ всегда определяет невырожденную инвариантную симметричную билинейную форму на соответствующих фробениусовых алгебрах — коммутативных ассоциативных алгебрах $A(u)$ в $N$-мерном векторном пространстве с базисом $e_{1}, \ldots, e_{N}$ и умножением (см. [6])

$$
\begin{gathered}
e_{i} * e_{j}=\eta^{k s} \frac{\partial^{3} \Phi}{\partial u^{s} \partial u^{i} \partial u^{j}} e_{k}, \\
\left\langle e_{i}, e_{j}\right\rangle=\eta_{i j}, \quad\left\langle e_{i} * e_{j}, e_{k}\right\rangle=\left\langle e_{i}, e_{j} * e_{k}\right\rangle,
\end{gathered}
$$

причем условие ассоциативности

$$
\left(e_{i} * e_{j}\right) * e_{k}=e_{i} *\left(e_{j} * e_{k}\right)
$$

в алгебрах $A(u)$ эквивалентно уравнениям (5.34). Напомним (см. [6]), что локально на любом фробениусовом многообразии на касательном пространстве в каждой точке имеется структура фробениусовой алгебры (7.5)-(7.7), определяемая некоторым решением уравнений ассоциативности (5.34) и гладко зависящая от точки (при этом на фробениусовых многообразиях требуется еще выполнение дополнительных условий, которые мы здесь не рассматриваем, грубо говоря, требуется наличие единицы и квазиоднородность). Таким образом, с каждым фробениусовым многообразием связаны нелокальные гамильтоновы операторы вида (7.1) и пучки (7.2).

Структурные потоки (см. [1,5]) нелокального гамильтонова оператора (7.1) имеют вид

$$
u_{t_{n}}^{i}=\eta^{i s} \frac{\partial^{3} \Phi}{\partial u^{s} \partial u^{n} \partial u^{k}} u_{x}^{k}
$$


Эти системы являются интегрируемыми бигамильтоновыми системами гидродинамического типа и совпадают с первичной частью иерархии Дубровина, построенной по любому решению уравнений ассоциативности в [6]. Условие коммутации структурных потоков (7.8) эквивалентно опять-таки уравнению ассоциативности (5.34).

Из рассмотрений предыдущего параграфа получаем, что по теореме Петерсона-Бонне любое решение $\Phi(u)$ уравнений ассоциативности (5.34) определяет в $2 N$-мерном псевдоевклидовом пространстве единственное с точностью до движений $N$-мерное подмногообразие с плоской нормальной связностью, с первой квадратичной формой $\eta_{i j} d u^{i} d u^{j}$ и вторыми квадратичными формами

$$
\omega_{n}(u)=\frac{\partial^{3} \Phi}{\partial u^{n} \partial u^{i} \partial u^{j}} d u^{i} d u^{j}
$$

задаваемыми потенциалом $\Phi(u)$. При этом сигнатура объемлющего псевдоевклидова пространства полностью определяется сигнатурой метрики $\eta_{i j}$. В этом случае уравнение ассоциативности совпадает и с уравнением Гаусса, и с уравнением Риччи вложенного подмногообразия. Таким образом, $N$-мерным фробениусовым многообразиям локально отвечает специальный класс плоских $N$-мерных подмногообразий с плоской нормальной связностью в $2 N$-мерном псевдоевклидовом пространстве.

Большое количество конкретных примеров фробениусовых многообразий и решений уравнений ассоциативности приведено в работе Дубровина [6]. Для экономии места мы не будем приводить здесь большого количества примеров и явного вида соответствующих гамильтоновых операторов, плоских подмногообразий и интегрируемых иерархий и рассмотрим в качестве иллюстрации только один простой пример из [6]. Пусть $N=3$, метрика $\eta_{i j}$ антидиагональна,

$$
\left(\eta_{i j}\right)=\left(\begin{array}{lll}
0 & 0 & 1 \\
0 & 1 & 0 \\
1 & 0 & 0
\end{array}\right)
$$

а функция $\Phi(u)$ имеет вид

$$
\Phi(u)=\frac{1}{2}\left(u^{1}\right)^{2} u^{3}+\frac{1}{2} u^{1}\left(u^{2}\right)^{2}+f\left(u^{2}, u^{3}\right) .
$$

В этом случае $e_{1}$ является единицей в соответствующей фробениусовой алгебре (7.5)-(7.7), а уравнение ассоциативности (5.34) для функции $\Phi(u)$ эквивалентно замечательному интегрируемому уравнению Дубровина

$$
\frac{\partial^{3} f}{\partial\left(u^{3}\right)^{3}}=\left(\frac{\partial^{3} f}{\partial\left(u^{2}\right)^{2} \partial u^{3}}\right)^{2}-\frac{\partial^{3} f}{\partial\left(u^{2}\right)^{3}} \frac{\partial^{3} f}{\partial u^{2} \partial\left(u^{3}\right)^{2}}
$$

для функции $f\left(u^{2}, u^{3}\right)$, связанному с квантовыми когомологиями проективной плоскости и классическими задачами исчислительной геометрии (см. [7]). Как 
показано автором в [8] (см. также [9]), уравнение (7.10) эквивалентно интегрируемой недиагонализуемой однородной системе гидродинамического типа

$$
\begin{gathered}
\left(\begin{array}{l}
a \\
b \\
c
\end{array}\right)_{u^{3}}=\left(\begin{array}{ccc}
0 & 1 & 0 \\
0 & 0 & 1 \\
-c & 2 b & -a
\end{array}\right)\left(\begin{array}{l}
a \\
b \\
c
\end{array}\right)_{u^{2}}, \\
a=\frac{\partial^{3} f}{\partial\left(u^{2}\right)^{3}}, \quad b=\frac{\partial^{3} f}{\partial\left(u^{2}\right)^{2} \partial u^{3}}, \quad c=\frac{\partial^{3} f}{\partial u^{2} \partial\left(u^{3}\right)^{2}} .
\end{gathered}
$$

В этом случае аффиноры нелокального гамильтонова оператора (7.1) имеют вид

$$
\left(w_{1}\right)_{j}^{i}(u)=\delta_{j}^{i}, \quad\left(w_{2}\right)_{j}^{i}(u)=\left(\begin{array}{ccc}
0 & b & c \\
1 & a & b \\
0 & 1 & 0
\end{array}\right), \quad\left(w_{3}\right)_{j}^{i}(u)=\left(\begin{array}{ccc}
0 & c & b^{2}-a c \\
0 & b & c \\
1 & 0 & 0
\end{array}\right) .
$$

\section{ЛитерАТУРА}

1. Ферапонтов E. B. Дифференциальная геометрия нелокальных гамильтоновых операторов гидродинамического типа. Функц. анализ и его прил., 25, вып. 3, 3749 (1991).

2. Дубровин Б. А., Новиков С. П. Гамильтонов формализм одномерных систем гидродинамического типа и метод усреднения Боголюбова-Уизема. Докл. АН СССР, 270, № 4, 781-785 (1983).

3. Мохов О. И., Ферапонтов Е. В. О нелокальных гамильтоновых операторах гидродинамического типа, связанных с метриками постоянной кривизны. УМН, 45, вып. 3, 191-192 (1990).

4. Magri F. A simple model of the integrable Hamiltonian equation. J. Math. Phys., 19, No. 5, 1156-1162 (1978).

5. Maltsev A. Ya., Novikov S. P. On the local systems Hamiltonian in the weakly nonlocal Poisson brackets. Phys. D, 156, Nos. 1-2, 53-80 (2001); arXiv: nlin.SI/0006030 (2000).

6. Dubrovin B. Geometry of 2D topological field theories. In: Integrable Systems and Quantum groups, Lecture Notes in Math., Vol. 1620, Springer-Verlag, Berlin, 1996, pp. 120-348; arXiv: hep-th/9407018 (1994).

7. Kontsevich M., Manin Yu. I. Gromov-Witten classes, quantum cohomology, and enumerative geometry. Comm. Math. Phys., 164, 525-562 (1994).

8. Mokhov O. I. Symplectic and Poisson geometry on loop spaces of manifolds and nonlinear equations. In: Topics in Topology and Mathematical Physics (S. P. Novikov, ed.), Amer. Math. Soc. Transl. Ser. 2, Vol. 170, Amer. Math. Soc., Providence, RI, 1995, pp. 121-151; arXiv: hep-th/9503076 (1995).

9. Мохов О. И. Симплектическая и пуассонова геометрия на пространствах петель гладких многообразий и интегрируемые уравнения. ИКИ, Москва-Ижевск, 2004.

Центр нелинейных исследований при Институте теоретической физики им. Л. Д. Ландау РАН e-mail:mokhov@mi.ras.ru,mokhov@landau.ac.ru
Поступило в редакцию 10 мая 2004 г. 